\section{Plasma motion near comet cores}

\author{
from D.J. Southwood
}

COMETARY fever is rising in the space science community with the launching of space probes to Comet Halley and the imminent encounter of the old ISEE 3 spacecraft (now renamed International Cometary Explorer) with Giacobini - Zinner. One group could be said to have stolen a march: on 27 December last year the West German spacecraft of an international mission was enveloped by what was heralded as the first artificial comet, a cloud of ionized barium released from the spacecraft itself, and observed from Earth as well. But, by the time of the third meeting of the AMPTE (Active Magnetospheric Particle Tracer Explorers) science team held in June at Thornbridge Hall, Derbyshire, doubts were arising about the appropriateness of the cometary epithet amongst the scientists from the three nations (United States, West Germany and United Kingdom) involved in the mission.

It had, of course, been realized that the release, made in the solar wind on the dawnside of Earth one hundred thousand kilometres above the Earth's surface, would throw no light on the cosmogonic problems that could only be resolved by a spacecraft encounter with a comet; but it was confidently believed that the solar wind deflection, cometary plasma tail formation and depletion processes would be mimicked. The initial images obtained from the ground and from aircraft of the ion cloud expansion, elongation and eventual dispersal (after about five minutes), only served to confirm that prejudice. So did the magnetic field measurements made on the German IRM (Ion Release Module) spacecraft and its nearby companion, the UKS (United Kingdom Satellite), in the immediate vicinity of the cloud. In particular UKS recorded clearly the 'draping' of the field over the cloud exactly as was expected. Now, as the detailed analysis is proceeding, second thoughts are occurring. Analysis is complex because both IRM and UKS carried a complement of field and plasma instrumentation and the teams are having to come to terms not only with the images obtained from afar but also with the in situ measurements of local plasma conditions in and near the cloud.

The dramatic evidence that the team is now coming to terms with concerns the relative motion of the ion cloud and the faint residue of non-ionized barium that can just be detected in some of the images. Simple ideas from cometary theory led one to expect the cloud to move away from the Sun. The reason is straightforward: momentum is absorbed from the solar wind which is moving radially away from the Sun. Gerhard Haerendel of the Max Planck Institut für Extraterrestrische Physik, the German project scientist, summarized the overall picture that is emerging. At the core is a very surprising result: the initial motion of the ion cloud formed at the core of the release was transverse to the solar wind direction. Where does the transverse momentum come from? It seems that it is due to a recoil effect from a stream of high speed ions leaking out of the cloud in the opposite sense. These ions later form the dominant component of the tail which ultimately extends thousands of kilometres away from the original hundredkilometre-radius cloud at the core of the release. Although dissatisfaction had been expressed by many about the simple cometary theory based on magnetohydrodynamics (MHD), the high-speed ions were unexpected.

It is important to note that all interactions take place in a medium that is collision-free: the mean free path is orders of magnitude larger than the cloud. The electromagnetic field structure that produces the observed effects is complicated and presents an exciting challenge to plasma physics. In some respects, the physics resembles that required to describe collisionless shocks but with the further complication that the cloud 'obstacle' to the solar wind is relatively small and evidently three dimensional - whereas shock theory is normally developed for

\section{Mathematics}

\section{from Ian Stewart}

ONE of the more romantic figures in mathematics is the young revolutionary Evariste Galois, who wrote down his ideas on the theory of equations the night before a fatal duel, ostensibly over a lady. $\mathrm{He}$ introduced what is now called the Galois group of an equation, which measures how symmetrical it is and contains information about its solutions. Ever since, mathematicians have wondered which groups can occur as Galois groups. John G. Thompson (J. Algebra 89, 437; 1984 ) has now found new ways to realize specific groups as Galois groups, including a remarkable 'sporadic simple group' known as the monster, because of its enormous size.

Galois showed that an equation can be solved by radicals - a formula involving nothing worse than $n$th roots - if, and only if, it has a restricted type of sym- planar structures. Shock scale lengths are short, however, and comparable with that of the cloud core, which is smaller than the magnetic (Larmor) gyration radii of either the solar wind ions $(300 \mathrm{~km})$ or the newly created barium ions $(3,000 \mathrm{~km})$. The ions behave as if they are unmagnetized. One consequence is that the obstacle has a high Hall conductivity, and therefore carries large currents perpendicular to any applied electric field. Such effects are negligible when MHD is valid. As often occurs in plasmas, it is when MHD breaks down that the really surprising physics is found. The momentum transfer between cloud and wind requires substantial electron heating as an intermediate process. Somehow a small fraction of the released ions manage to tap into the energy carried by the heated solar wind electrons and this gives rise to the curious effect reported.

Despite these comments, the results are probably still relevant to comets. Cometary cores can be small compared to the Larmor orbit scale of the massive ions believed to make up the cometary plasma environment. The relatively scant notice so far taken of such facts may be due to theory never before having to come to terms with the reality of in situ measurements. In the broader context the results on anomalous acceleration and heating of plasmas are going to be of importance not only to plasma physics but also for astrophysics. In these experiments we see a cosmic process writ small. The team have a second opportunity to test their ideas as another barium release took place outside the magnetosphere on the duskside of Earth on 18 July.

D.J. Southwood is in the Space Physics Group, Blackett Laboratory, Imperial College, London SW7 2AZ, UK.

\title{
The duellist and the monster
}

metry: its Galois group must be 'soluble'. For example, the general equation of the fifth degree has a Galois group containing all 120 different ways of permuting its five roots. This is insoluble, hence the quintic equation cannot be solved by radicals. The group is known to be insoluble because it contains within it a smaller 'simple' group $A_{5}$ with only 60 elements. (A simple group is one that cannot be broken down into smaller groups in a particular way.)

It seems to have been Emmy Noether, the most famous woman mathematician, who first asked which groups could occur as Galois groups. The most likely answer, "all of them", has never been proved or disproved. Over a period of time, various partial answers were obtained, notably by Igor Safarevič, culminating in a proof that every soluble group is a Galois group. The 